Chiu Fan Lee is in the Department of

Bioengineering, Faculty of Engineering,

Imperial College London, London SW7 2AZ, UK.

e-mail: c.lee@imperial.ac.uk

1. Israelachvili, J. N. Intermolecular and Surface Forces 3rd edn (Elsevier, 2010)

2. Riback, J. A. et al. Nature 581, 209-214 (2020)

3. Banani, S. F., Lee, H. O., Hyman, A. A. \& Rosen, M. K. Nature Rev. Mol. Cell Biol. 18, 285-298 (2017).
4. Feric, M. et al. Cell 165, 1686-1697 (2016).

5. Mitrea, D. M. et al. elife 5, e13571 (2016).

6. Shin, Y. \& Brangwynne, C. P. Science 357, eaaf4382 (2017)

7. Olson, M. O. J., Dundr, M. \& Szebeni, A. Trends Cell Biol. 10, 189-196 (2000).

8. Berry, J., Brangwynne, C. P. \& Haataja, M. Rep. Prog. Phys. 81, 046601 (2018).

9. Weber, C. A., Zwicker, D., Jülicher, F. \& Lee, C. F. Rep. Prog Phys. 82, 064601 (2019).

This article was published online on 6 May 2020.

\title{
Airborne particles might grow fast in cities
}

\section{Hugh Coe}

Nanoscale particles have been observed to form and grow

in the atmospheres of many cities, contradicting our understanding of particle-formation processes. Experiments now reveal a possible explanation for this mystery. See p.184

On page 184 , Wang et al. ${ }^{1}$ report observations of the rapid growth of newly formed atmospheric particles through the condensation of ammonium nitrate under conditions typical of many urban environments in wintertime. The observations were made in a chamber in a laboratory, but the authors convincingly argue that similar conditions can occur transiently in megacities. The findings fill a major gap in our knowledge of particle growth rates in cities.

Particulate matter is a key factor in the air quality of many of the world's megacities because it has been directly linked to multiple non-communicable diseases (see go.nature. com/2w49q1t). It also substantially affects regional climate through its interactions with solar radiation and clouds ${ }^{2}$. Particle-formation processes are important in the air above large cities because they replenish the particle population, determine the total particle-number concentration and can act as 'seeds' for cloud formation. We therefore need to know how particles form and grow in order to predict the effects of particulate matter on health and regional climate.

Although our knowledge of particle formation has improved over the past few years ${ }^{3,4}$, our understanding of the early stages of particle growth - particularly the crucial step in which an initial cluster of molecules grows large enough to become an actual particle - cannot explain why new particles form in megacity environments ${ }^{5}$. The persistence of newly formed clusters depends on the ratio of the condensation sink (the rate at which vapour and clusters are scavenged by pre-existing particles) to the growth rate of the clusters ${ }^{3}$. In the real world, both of these quantities depend on the particle-size distribution.

The condensation sink can be derived directly from the particle-size distribution. However, the growth rate is commonly determined by monitoring how clusters grow over time, typically in the size range between 1 and 10 nanometres. This method assumes that the environmental factors that affect cluster growth are uniform throughout a given

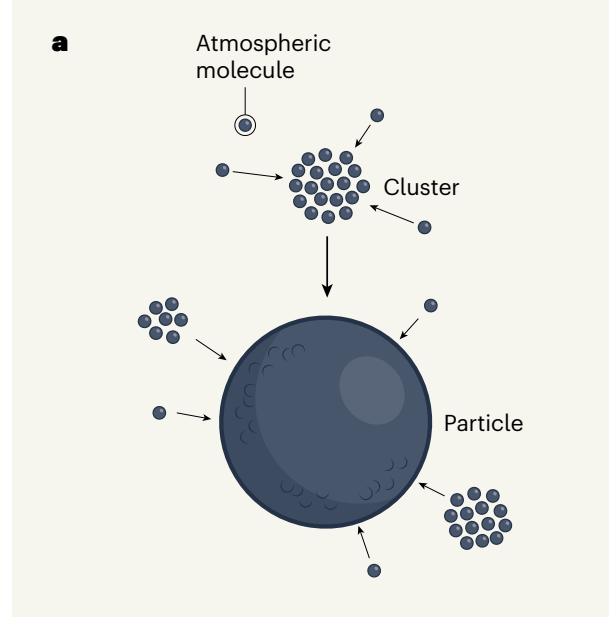

Figure 1 The growth and formation of atmospheric particles. a, Small clusters of atmospheric molecules can gradually accumulate more molecules until they form stable particles. However, other particles in the atmosphere can scavenge the available vapour, limiting cluster growth, or even scavenge whole clusters. The concentration of particles in urban environments is high, which means that any clusters or vapour would be expected to be scavenged by existing particles before they form stable particles themselves. Yet the observed rate of new-particle formation is surprisingly high in megacities. b. Wang et al. ${ }^{1}$ report that clusters can grow rapidly by accumulating ammonium nitrate (which forms from ammonia and nitric acid molecules) under conditions known to occur in megacities in winter. This allows the clusters to reach stable particle sizes before they are scavenged by other particles - and might explain the high particle-formation rates observed in urban areas. b

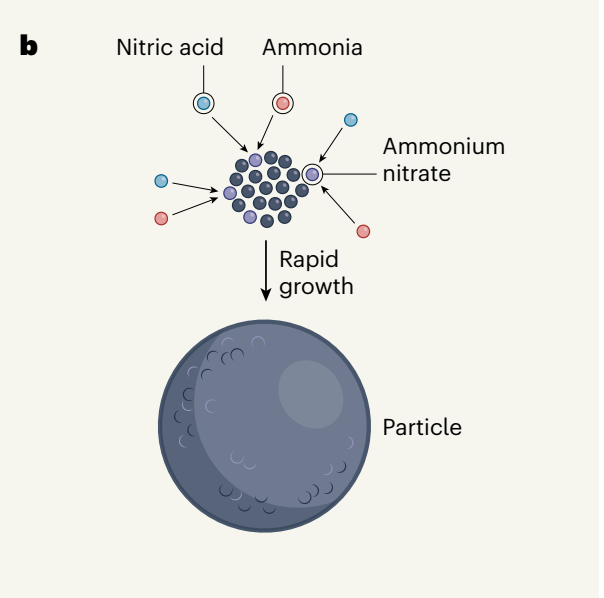

region, and it has worked well in describing particle-growth behaviour in rural environparticle growth in cities ${ }^{5}$.

The particle loading of air in urban environments can be greater than 500 micrograms per cubic metre (ref. 6), whereas that of rural or remote environments is usually less than $5 \mu \mathrm{g} \mathrm{m}^{-3}$ (ref. 7). Newly formed clusters in cities must therefore rapidly scavenge vapour or combine with other clusters so that they can grow large enough for the rates at which they are themselves scavenged to be reduced Fig. 1a), and therefore survive to become persistent, larger particles. Given that only a few times greater than those in remote environments, it is hard to understand how newly formed particles can reach diameters of $10 \mathrm{~nm}$ or more in urban areas - but such growth seems to be widespread in megacities in wintertime.

Wang et al. investigated this issue by carrying out a set of chamber experiments that reproduced atmospheric conditions typical megacity, focusing on the behaviour of component of urban winter- and springtime particulate matter ${ }^{8}$, but has not been thought o have a major role in particle formation.

Ammonium nitrate exists in a temperature-dependent equilibrium with gaseous ammonia and nitric acid, and this equilibrium the authors observed that ammonium nitrate rapidly condenses onto newly formed clusters at temperatures below $5^{\circ} \mathrm{C}$ (Fig. 1b). This is 


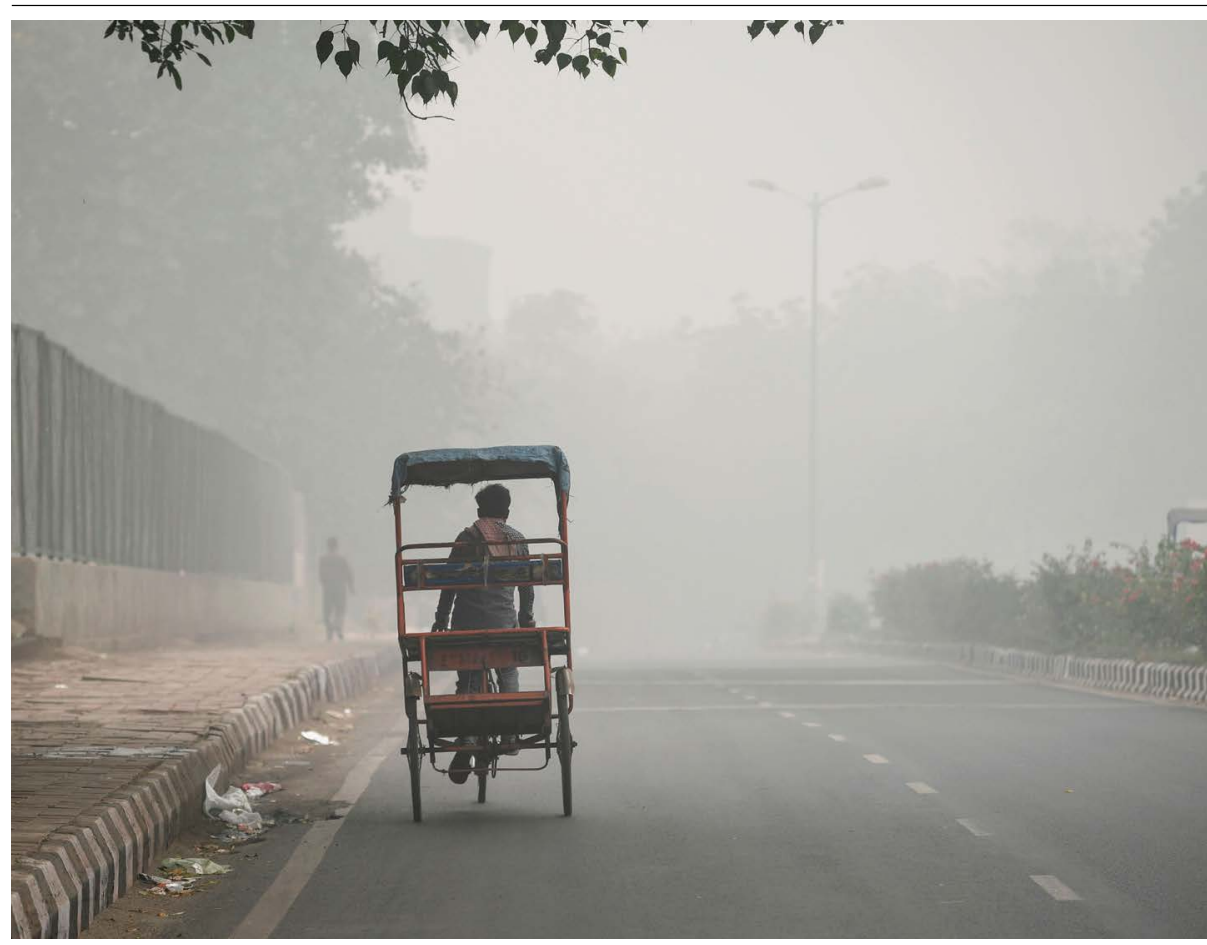

Figure 2 | Heavy smog in Delhi during winter 2019. Severe wintertime pollution events in megacities can produce atmospheric conditions similar to those reported by Wang et al. ${ }^{1}$ to cause rapid growth of atmospheric particles.

because the atmospheric concentrations of ammonia and nitric acid vapours at these temperatures can exceed their equilibrium values. To put it another way, when the ratio of the concentration of these gases to their concentration at equilibrium (the saturation ratio) under the same environmental conditions is greater than 1 , rapid condensation takes place.

Crucially, the observed rapid condensation accelerates particle growth. The particle growth rates at $-10^{\circ} \mathrm{C}$ were 200 times faster than those at $+5^{\circ} \mathrm{C}$, for the same gas concentrations of ammonia and nitric acid. The growth rates at cold temperatures are much higher than those previously derived from field observations in urban areas.

By measuring the composition of vapours and particles using an array of advanced mass spectrometers, Wang and colleagues showed that ammonium nitrate does not participate in particle formation at temperatures above $-15^{\circ} \mathrm{C}$. Particle formation instead proceeds through a well-recognized pathway involving ammonia and sulfuric acid ${ }^{9}$; rapid growth through ammonium nitrate condensation begins to occur once a threshold cluster size has been reached. However, the authors report that new particles can form directly from ammonium nitrate at temperatures below $-15^{\circ} \mathrm{C}$. The authors speculate that this process could occur in the humid air outflows at the top of convective tropical clouds.

The authors show that the critical size at which ammonium nitrate starts to induce rapid growth depends on the saturation ratio of the ammonia-nitric acid system.
Furthermore, once a particle has reached that size, it continues to grow rapidly because the equilibrium concentration of ammonia and nitric acid above ammonium nitrate is much lower for larger particles. This growth occurs in much the same way that a liquid cloud forms on particles called condensation nuclei, which grow rapidly as soon as the saturation ratio of water exceeds 1 .

So, how representative of the real world are these experimental observations, and what do

\section{“This work provides key knowledge that will inform air-quality policy as the chemical composition of urban atmospheres changes in the future."}

they tell us about real urban environments? The mixing ratios of ammonia and nitric acid in the experiments are typical of those of many urban environments and are often greatly exceeded in some megacities. Moreover, in many places, such as Beijing or Delhi (Fig. 2), severe air-pollution events involving high concentrations of ammonia and nitric acid occur often, mostly in wintertime when the daytime temperatures are at, or below, $5^{\circ} \mathrm{C}$ (see ref. 10 , for example).

However, air must become supersaturated with ammonia and nitric acid before clusters can grow through ammonium nitrate condensation. Wang et al. convincingly argue that localized supersaturation of these gases is likely in many cities because the environment is heterogeneous. For example, the emission sources vary widely, and the flow of emissions around buildings, in street canyons and as a result of traffic movement combine to generate substantial gradients of concentration. The temperature in cities also often varies by several degrees over distances of a few metres to a few tens of metres, because of direct heating or shadowing from buildings, and because different surfaces absorb and reflect heat differently. These temperature variations can alter the saturation ratio of ammonia and nitric acid sufficiently for rapid condensation to occur.

Wang and colleagues calculate that the rapid condensation of ammonia and nitric acid occurs on timescales of several minutes in their experiments. The temperature heterogeneities observed in cities are sustained for similar timescales across various distances, potentially allowing clusters to grow to more-stable sizes at which further mass can be added to grow the particles. In other words, the new findings might explain why the initial stages of particle growth can be so fast in cities. Previously calculated cluster-growth rates in cities were averaged over space and time, and therefore did not capture this heterogeneity.

It will be extremely challenging to demonstrate that rapid ammonium nitrate condensation occurs in the real atmosphere, but the concept is very persuasive. Numerous semi-volatile organic compounds in the atmosphere might well have a similar role in particle growth. More broadly, Wang and colleagues' work provides key knowledge that will inform air-quality policy as the chemical composition of urban atmospheres changes in the future. Most notably, sulfur dioxide emissions are being reduced across many cities. This makes it increasingly likely that urban pollution will be dominated by emissions of nitrogen oxide (a precursor of nitric acid) from road traffic and by ammonia from agriculture for the coming decade or more.

Hugh Coe is in the Department of Earth and Environmental Sciences, University of Manchester, Manchester M13 9PL, UK. e-mail: hugh.coe@manchester.ac.uk

\footnotetext{
. Wang, M. et al. Nature 581, 184-189 (2020).

2. Seinfeld, J. H. Proc. Natl Acad. Sci. USA 113, 5781-5790 (2016).

3. Yao, L. et al. Science 361, 278-281 (2018).

4. Ehn, M. et al. Nature 506, 476-479 (2014).

5. Kulmala, M., Kerminen, V.-M., Petäjä, T., Ding, A. J. \& Wang, L. Faraday Discuss. 200, 271-288 (2017).

6. Chen, Y. et al. Atmos. Environ. X 5, 100052 (2020).

7. Van Dingenen, R. et al. Atmos. Environ. 38, 2561-2577 (2004).

8. Young, D. E. et al. Atmos. Chem. Phys. 15, 6351-6366 (2015).

9. Kirkby, J. et al. Nature 476, 429-433 (2011)

10. Huang, R.-J. et al. Nature 514, 218-222 (2014).
} 